\title{
The SLIM1 transcription factor regulates arsenic sensitivity in Arabidopsis thaliana
}

\author{
Timothy Jobe ${ }^{1}$, Qi Yu ${ }^{2}$, Felix Hauser ${ }^{2}$, Qingqing Xie ${ }^{3}$, Yuan Meng ${ }^{2}$, Tim Maassen ${ }^{1}$, \\ Stanislav Kopriva ${ }^{4}$, and Julian Schroeder ${ }^{5}$ \\ ${ }^{1}$ University of Cologne Department of Biologie \\ ${ }^{2}$ University of California San Diego \\ ${ }^{3}$ Salk Institute \\ ${ }^{4}$ University of Cologne \\ ${ }^{5}$ Division of Biological Sciences, Cell and Developmental Biology Section
}

December 28, 2020

\begin{abstract}
The transcriptional regulators of arsenic-induced gene expression remain largely unknown; however, arsenic exposure rapidly depletes cellular glutathione levels increasing demand for thiol compounds from the sulfur assimilation pathway. Thus, sulfur assimilation is tightly linked with arsenic detoxification. To explore the hypothesis that the key transcriptional regulator of sulfur assimilation, SLIM1, is involved in arsenic-induced gene expression, we evaluated the response of slim1 mutants to arsenic treatments. We found that slim1 mutants were sensitive to arsenic in root growth assays. Furthermore, arsenic treatment caused high levels of oxidative stress in the slim1 mutants, and slim1 mutants were impaired in both thiol and sulfate accumulation. We also found enhanced arsenic accumulation in the roots of slim1 mutants. Furthermore, microarray analyses identified genes from a highly co-regulated gene cluster (the O-acetylserine gene cluster), as being significantly upregulated in the slim1-1 mutant background in response to arsenic exposure. The present study identified the SLIM1 transcription factor as an important component in arsenic-induced gene expression and arsenic tolerance. Our results suggest that the severe arsenic sensitivity of the slim1 mutants is a result of both altered redox status as well as mis-regulation of key genes.
\end{abstract}

\section{Hosted file}

S1_Manuscript_PCE_Submit.pdf available at https://authorea.com/users/385835/articles/501057the-slim1-transcription-factor-regulates-arsenic-sensitivity-in-arabidopsis-thaliana 